\title{
Current updates on clinical management of COVID-19 infectees: a narrative review
}

\author{
Muhammad Taqi1* (D), Syed Faraz ul Hassan Shah Gillani1 (D), \\ Mishaal Tariq² (10, Zulfiqar Ali Raza ${ }^{3 *} \mathbb{1}$, Mobeen Zaka Haider ${ }^{1}$
}

\section{INTRODUCTION}

The severe acute respiratory syndrome coronavirus-2 (SARS-CoV-2) has emerged the current public health crisis, thus resulting in medical emergencies worldwide. This virus likely originated from bats, through an unknown intermediary vector, and then transmitted to humans in Wuhan city of China in December 2019 ${ }^{1}$. The virus was designated as a novel coronavirus (2019-nCOV) by WHO and then widely used as coronavirus disease 2019 (COVID-19) ${ }^{2}$. The prevalence and severity of coronavirus depend on several factors such as viral serotypes, tropics, and genetic makeup of local people and also on the pandemic management protocols and the medical services $^{3}$. This study aims to narrate and analyze the available key guidelines and recommendations to manage the COVID-19 pandemic as no study is available until now covering all aspects of COVID-19 from clinical features, diagnosis to management, and healthcare guidelines. Thus, a comprehensive approach needs to be addressed to minimize the confusion regarding the treatment and management of COVID-19 patients.

\section{Clinical features}

The clinical symptoms of COVID-19 infection are fever, productive cough, myalgia, fatigue, sputum expectoration, dyspnea, arthralgia, headaches, sore throat, chills, nausea, vomiting, diarrhea, pleuritic chest pain, nasal congestion, palpitations, chest tightness, and hyposmia. Complications are acute respiratory distress syndrome (ARDS), acute myocardial injury, raised troponin I levels, myocardial infarction, sudden cardiac arrest, secondary bacterial infections, sepsis, multiorgan failure, lymph histiocytosis, and the cytokine storm syndromes.
Deaths are more prevalent in elderly patients and already medically ill patients like malignancy and interstitial lung disease ${ }^{4}$. The COVID-19 patients with diabetes are at higher risk of severe pneumonia due to the release of certain enzymes, the vicious cycle of inflammatory cascades, and hypercoagulable states $^{5}$. Similarly, the patients with chronic obstructive pulmonary disease (COPD) may far seriously affect ${ }^{6}$.

The diagnostic indication of COVID-19 varies from patient to patient depending on patients' medical history, clinical examination, medical baseline, and the test's specificity. The realtime polymerase chain reaction (RT-PCR) expressed 60-97\% sensitivity. However, some cases have been reported to exhibit negative RT-PCR results whereas high-resolution CT (HR-CT) scans turned out to be positive for the same patients. So the inclusion of radiological features in diagnostic criteria increases the specificity of testing 7 . Few radiographs and CT images showing consolidation and thickened pleura are shown in Figure 1.

The drugs that have potential activity against coronavirus include remdesivir, ritonavir, lopinavir, lopinavir/ritonavir combined with interferon- $\beta$ (INF- $\beta$ ), monoclonal antibodies, and antimalarial drug (i.e., hydroxychloroquine) ${ }^{8}$. Anticoagulant therapy with low molecular weight heparin (LMWH) appears to be associated with a better prognosis in treated patients vs. nontreated ones concerning mortality ( 40.0 versus $64.2 \%, \mathrm{p}=0.029)^{9}$.

\section{Clinical management}

If patients have been screened as positive for common flu-like symptoms, a surgeon should consider delaying any surgical procedures until the patient stabilizes, and if the surgery is

\footnotetext{
'King Edward Medical University, Mayo Hospital, Department of Orthopedic Surgery - Lahore, Pakistan.

${ }^{2}$ King Edward Medical University, Mayo Hospital, Department of Anesthesia - Lahore, Pakistan.

${ }^{3}$ National Textile University, Department of Applied Sciences - Faisalabad, Pakistan.

*Corresponding author: dr.taqi227@gmail.com, zarazapk@yahoo.com

Conflicts of interest: the authors declare there is no conflicts of interest. Funding: none.

Received on June 14, 2021. Accepted on June 27, 2021.
} 


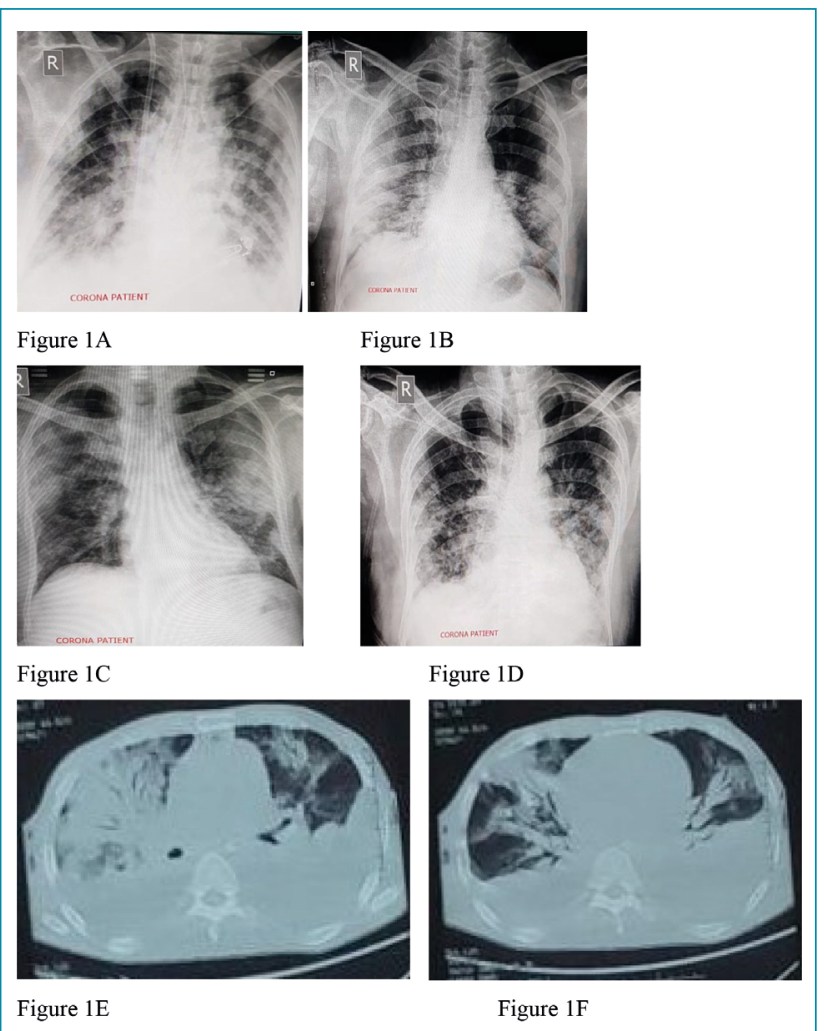

Figure 1. Representative radiographs: (A) honeycomb appearance (ARDS), (B) bronchopneumonia, (C) hyperinflation with consolidation, and (D) hyperemia with basal consolidation; and the computed tomography scan images at axial view showing (E) consolidation with air bronchogram and (F) fibrotic band and pneumonic patches.

unavoidable, such patients must undergo urgent RT-PCR testing $^{10}$. The American Academy of Orthopedic Surgeons (AAOS) recommends patients' ELIZA or PCR testing for COVID-19 $72 \mathrm{~h}$ before surgery or on the day of surgery, and the healthcare workers (HCWs) before performing any surgical procedures. The following guidelines should be followed while treating/ operating patients during this pandemic ${ }^{11}$ :

1. Resuscitate the patient, rule out all other injuries via primary survey;

2. Do secondary survey as there are high chances of missed injuries in light of COVID suspicion;

3. Whenever possible, manage conservatively and keep the patients in isolation wards;

4. Provide masks to patients and attendants, and minimize patients' and attendants' mobility;

5. Expedite the process of operation and discharge to lessen the load over the health system, and these patients should be attended by separate team surgeons;

6. Keep follow-up of outdoor patients in a separate area for dressing, suture removal, and plaster removal;
7. Manage the patient conservatively whenever possible, and preventive measures must be followed at every level;

8. There should be separate triage room and dedicated COVID operating room with trained staff;

9. Maintain negative pressure ventilation in operation theaters;

10. Minimize operation time and blood spillage;

11. Postoperatively, the patient should be shifted to COVID wards and discharged only after COVID results are negative;

12. Special care must be taken during the hospital stay to wound dressing, physiotherapy, bedsores, and deep vein thrombosis (DVT) prevention; and

13. There should be proper disposal of surgical waste. The clinical management summary of COVID-19 patients in various departments is illustrated in Figure 2.

The lungs are the primary organ affected by COVID-19 and transmission through aerosols, while thoracic surgery makes it a high-risk procedure for the HCW. Thoracic surgery is significantly associated with high mortality rates in COVID19-infected patients. It had been concluded that COVID-19 patients undergoing thoracic operation are associated with a poor prognosis, especially for those who are suffering from COPD. However, comprehensive protective measures are required to prevent and control COVID infection ${ }^{12}$. Thereby, the following precautions are necessary:

1. Perform the procedure in an isolated negatively ventilated room;

2. Limit the number of participants and time of surgery;

3. Make a small incision and place an air seal tie around the tube;

4. Use high-efficiency particulate air (HEPA) filters on suction equipment; and

5. Avoid entry into room $10 \mathrm{~min}$ after the surgery due to the persistence of droplets into the $\mathrm{air}^{13}$.

Respiratory management has a key role in COVID-19 treatment. Apart from the abovementioned treatment guidelines, certain other steps are deemed necessary while treating patients with pulmonary function tests (PFTs) is required for assessing asthma and chronic pulmonary disorders. For that purpose, a patient under investigation needs to blow into a peak flow meter that may increase the risk of aerosol spreading. It is therefore strongly recommended that the PFT procedures for the suspected or confirmed cases of COVID-19 may be delayed during this pandemic if it is not imperative. Disposable $99 \%$ effective filtering devices should be used during the PFTs if deemed necessary. More attention is required on the disinfecting and sterilization of the equipment used using PFTs ${ }^{14}$. A passive mobilization of 


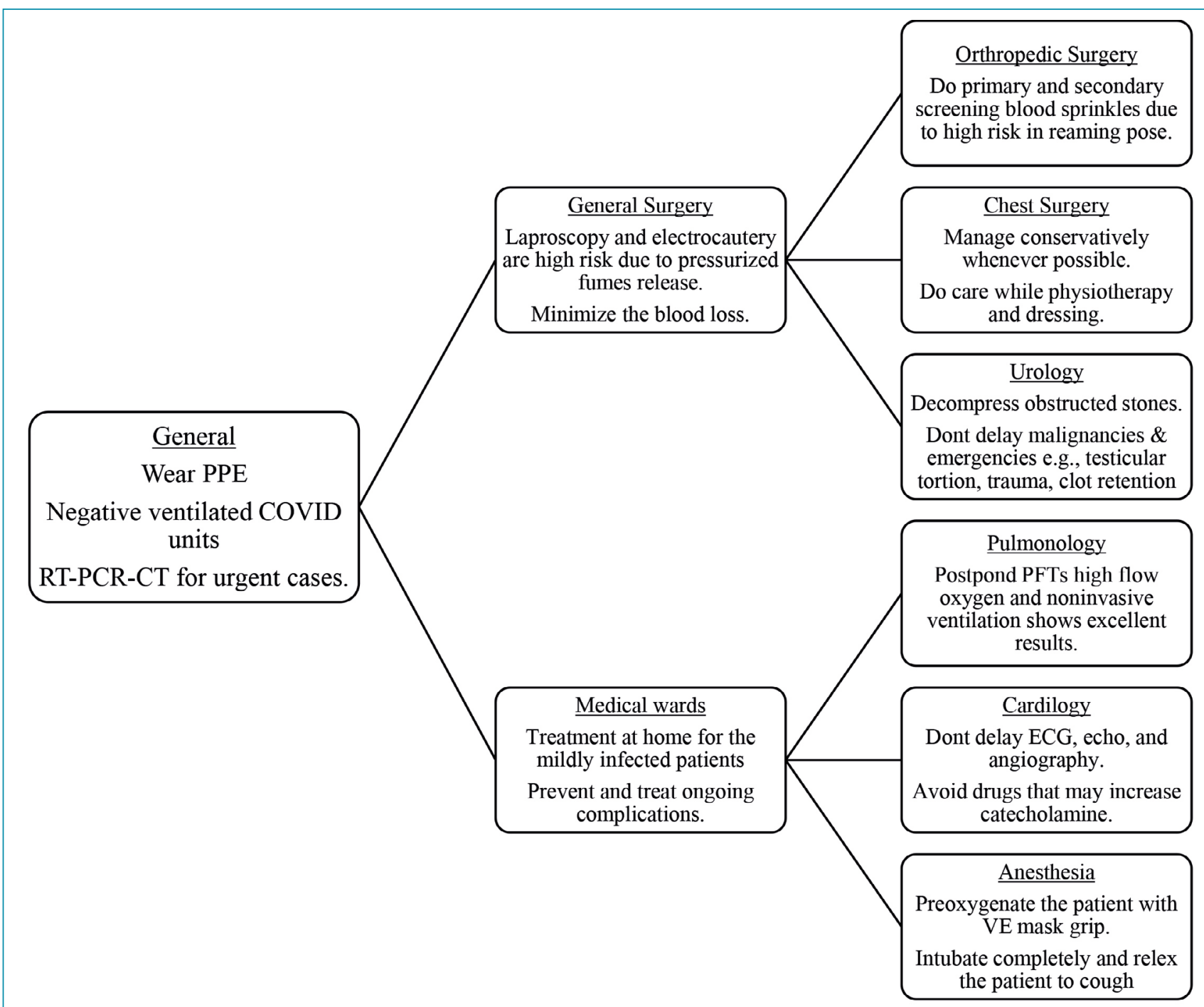

PPE: personal protection equipment; RT-PCR-CT: reverse transcriptase polymerase chain reaction-computarized tomography; PFTs: pulmonary function tests; ECG: electrocardiogram.

Figure 2. Clinical management summary of COVID-19 patients in various departments.

the patient is necessary to prevent skin lesions and bed sores. In spontaneous breathing patients, variation in position could modify the perfusion/ventilation ratio. Airway clearance is required only when it is indispensable. The patients should wear a face mask even during high nasal flow (HNF) oxygen therapy, and closed suctioning circuits are preferable when compared with endotracheal suctioning while disconnecting the circuit ${ }^{15}$.

In the anesthesiology, the rapid sequence induction (RSI) approach must be considered in the following steps:

1. Preoxygenate the patient with a well-fitting mask for 4-5 min;

2. In the distressed patients, the RSI may result in hypoxia so a delayed sequence tracheal intubation (DSTI) technique may be the best option;
3. In the case of cardiovascular instability or DSTI, $1-2 \mathrm{mg} / \mathrm{kg}$ ketamine is used for the induction of anesthesia;

4. Before proceeding to tracheal intubation, ensure full neuromuscular blockade and maintain patency through the guided airway;

5. Using two-person, two-handed technique with a VE-grip is effective in COVID-19-infected patients to suppress the dissemination of the virus;

6. A video laryngoscope is a correct option to be utilized;

7. Nasogastric tube may be required after tracheal intubation and ventilation to minimize the complications; and

8. Collect lower respiratory tract sample if COVID diagnosis is not confirmed. During anesthesia, drugs to minimize coughing such as dexmedetomidine, lidocaine, 
and opioids could be administered ${ }^{16}$. An overview of COVID-19 spread and management strategies is expressed in Figure 3.

\section{DISCUSSION}

The COVID-19 infection has inspired us to revisit all infection management strategies. Some drugs indicated effective in mild cases were found to be ineffective in severe cases. This occurred with lopinavir/ritonavir and other antiretrovirals in the clinical outcomes of severe cases, which might partly be due to the variable severity of the disease, viral load, different underlying disease, and clinical presentations ${ }^{17}$. Another cohort study included 102 adult patients; among patients who survived, they were younger and less likely to suffer from comorbidities. They suffered the least from complications and were less likely to require admission to the intensive care unit. There was no marked difference in drug treatment rates between the survival and non-survival groups. The trials showed that there were no differences in mortality among those who did or did not receive antimicrobial or glucocorticoid drug treatment ${ }^{18}$. Evidence was collected after the trial of remdesivir that the patient started improving on the 8th day of admission. The self-defense mechanism plays a key role in fighting against the coronavirus. The COVID-19 disease is self-limiting but it may persist for weeks due to complications and secondary infections developing after the disease $^{19}$. The antiviral activity is required against viral replicating enzymes, protein-synthesizing enzymes, assembly proteins, and entry receptors. Studies have shown that some natural products inhibit viral entry, replication, transcription, and translation limiting viral virulence and the spread of disease. The natural products have antiviral activity in the nanomolar concentration, e.g., curcumin, homoharringtonine, lycorine, Silvestre L, ouabain, tylophorine, and 7-methoxycryptopleurine, and silver nanoparticles ${ }^{20}$. These could further lead to drug development on their own or as a template. Besides, the major constituents of some common dietary supplements with anti-coronavirus activity may be used to boost the body's immunity against

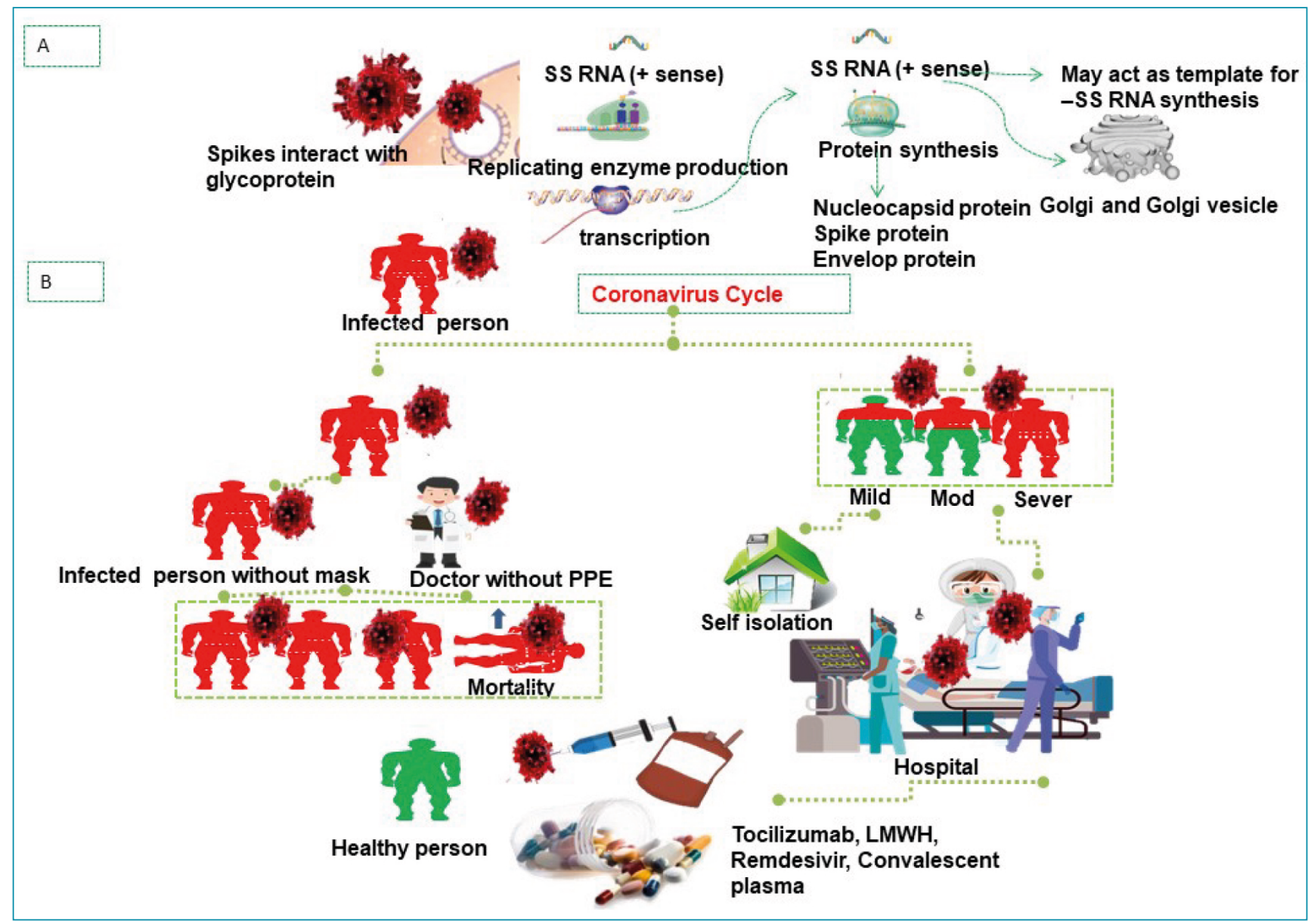

Figure 3. An overview of COVID-19 spread and management strategies: (A) the replication cycle of the corona virus. (B) the spread of the virus and COVID-19 management options. 
coronavirus $^{21}$. The main factor leading to pulmonary dysfunction in SARS survivors after recovery was pulmonary fibrosis, declining the quality of life. Extensive epidemiological, current clinical evidence supports the possibility that COVID-19 patients may face pulmonary fibrosis after the resolution of the coronavirus. Thus, some patients recovering from this infection may have diseased lungs and low quality of life when compared with a pre-pandemic state ${ }^{22}$. Further research is required for clinical trials, especially for developing the vaccine, this is the only way to get rid of this.

\section{CONCLUSION}

COVID-19 is the greatest pandemic health crisis of mankind. This study is designated to explain available guidelines and recommendations in major medical fields to minimize the risk of error. The clinical data of different drugs have been published, but large randomized control trials (RCTs) and cohort studies are required to prove the definitive role of anti-COVID drugs. Following clinical and practical guidelines, the dissemination of the COVID among HCWs and patients can be minimized as there is also an imbalance of demand and supply of personal protective equipment. Besides preventive measures, the COVID status of the patient needs to be documented in elective cases/ nonemergency cases. Keeping in mind the COVID load in the general public, both morbidity and mortality of HCW defer all unnecessary procedures and follow recommendations to make a safe way through this pandemic.

\section{AUTHORS' CONTRIBUTIONS}

MT: Investigation, Methodology, Writing - original draft. FHSG: Supervision. MT: Data curation. ZAR: Project administration, and Writing - review \& editing. MZH: Formal analysis.

\section{REFERENCES}

1. Zhu N, Zhang D, Wang W, Li X, Yang B, Song J, et al. A novel coronavirus from patients with pneumonia in China, 2019. N Engl J Med. 2020;382(8):727-33. https://doi.org/10.1056/ NEJMoa2001017

2. Li Q, Guan X, Wu P, Wang X, Zhou L, Tong Y, et al. Early transmission dynamics in Wuhan, China, of novel coronavirusinfected pneumonia. N Engl J Med. 2020;382(13):1199-207. https://doi.org/10.1056/NEJMoa2001316

3. Tang N, Bai H, Chen X, Gong J, Li D, Sun Z. Anticoagulant treatment is associated with decreased mortality in severe coronavirus disease 2019 patients with coagulopathy. J Thromb Haemost. 2020;18(5):1094-9. https://doi.org/10.1111/ jth. 14817

4. Zhou F, Yu T, Du R, Fan G, Liu Y, Liu Z, et al. Clinical course and risk factors for mortality of adult inpatients with COVID-19 in Wuhan, China: a retrospective cohort study. Lancet. 2020;395(10229):1054-62. https://doi.org/10.1016/S01406736(20)30566-3

5. Guo W, Li M, Dong Y, Zhou H, Zhang Z, Tian C, et al. Diabetes is a risk factor for the progression and prognosis of COVID19. Diabetes Metab Res Rev. 2020;31:e3319. https://doi. org/10.1002/dmrr.3319

6. Javanmardi F, Emami A, Pirbonyeh N. Global view of urinary tract drug-resistant infections in pregnant women: a systematic review and meta-analysis. SSRN. 2019. https://doi.org/10.2139/ ssrn. 3458514

7. Ding Q, Lu P, Fan Y, Xia Y, Liu M. The clinical characteristics of pneumonia patients coinfected with 2019 novel coronavirus and influenza virus in Wuhan, China. J Med Virol. 2020;92(9):154955. https://doi.org/10.1002/jmv.25781

8. Ye Q, Wang B, Mao J, Fu J, Shang S, Shu Q, et al. Epidemiological analysis of COVID-19 and practical experience from China. J Med Virol. 2020;92(7):755-69. https://doi.org/10.1002/ jmv.25813
9. Liu J, Li J, Arnold K, Pawlinski R, Key NS. Using heparin molecules to manage COVID-2019. Res Pract Thromb Haemost. 2020;4(4):518-23. https://doi.org/10.1002/rth2.12353

10. Forrester JD, Nassar AK, Maggio PM, Hawn MT. Precautions for operating room team members during the COVID-19 pandemic. J Am Coll Surg. 2020;230(6):1098-101. https:// doi.org/10.1016/j.jamcollsurg.2020.03.030

11. Neradi D, Hooda A, Shetty A, Kumar D, Salaria AK, Goni V. Management of orthopedic patients during COVID-19 pandemic in India: a guide. Indian J Orthop. 2020;54(3):402-7. https:// doi.org/10.1007/s43465-020-00122-6

12. Li YK, Peng S, Li LQ, Wang Q, Ping W, Zhang N, et al. Clinical and transmission characteristics of Covid-19-a retrospective study of 25 cases from a single thoracic surgery department. Curr Med Sci. 2020;40(2):295-300. https://doi.org/10.1007/ s11596-020-2176-2

13. Pieracci FM, Burlew CC, Spain D, Livingston DH, Bulger EM, Davis KA, et al. Tube thoracostomy during the COVID-19 pandemic: guidance and recommendations from the AAST Acute Care Surgery and Critical Care Committees. Trauma Surg Acute Care Open. 2020;5(1):e000498. https://doi. org/10.1136/tsaco-2020-000498

14. Task Force of Pulmonary Function Testing and Clinical Respiratory Physiology, Chinese Association of Chest Physicians; Pulmonary Function Testing Group, Respiratory Therapeutics Group, Chinese Thoracic Society. Expert consensus on pulmonary function testing during the epidemic of coronavirus disease 2019. Zhonghua Jie He He Hu Xi Za Zhi. 2020;43(4):302-7. https://doi.org/10.3760/cma.j.cn112147-20200225-00175

15. Lazzeri M, Lanza A, Bellini R, Bellofiore A, Cecchetto S, Colombo A, et al. Respiratory physiotherapy in patients with COVID-19 infection in acute setting: a position paper of the Italian Association of Respiratory Physiotherapists (ARIR). Monaldi Arch Chest Dis. 2020;90(1). https://doi.org/10.4081/ monaldi.2020.1285 
16. Cook TM, El-Boghdadly K, McGuire B, McNarry AF, Patel A, Higgs $A$. Consensus guidelines for managing the airway in patients with COVID-19: guidelines from the Difficult Airway Society, the Association of Anaesthetists the Intensive Care Society, the Faculty of Intensive Care Medicine and the Royal College of Anaesthetists. Anesthesia. 2020;75(6):785-99. https://doi.org/10.1111/anae.15054

17. Ford N, Vitoria M, Rangaraj A, Norris SL, Calmy A, Doherty M. Systematic review of the efficacy and safety of antiretroviral drugs against SARS, MERS or COVID-19: initial assessment. J Int AIDS Soc. 2020;23(4):e25489. https://doi.org/10.1002/ jia2.25489

18. Cao J, Tu WJ, Cheng W, Yu L, Liu YK, Hu X, et al. Clinical features and short-term outcomes of 102 patients with coronavirus disease 2019 in Wuhan, China. Clin Infect Dis. 2020;71(15):748-55. https://doi.org/10.1093/cid/ ciaa243
19. Cao YC, Deng QX, Dai SX. Remdesivir for severe acute respiratory syndrome coronavirus 2 causing COVID-19: an evaluation of the evidence. Travel Med Infect Dis. 2020;35:101647. https:// doi.org/10.1016/j.tmaid.2020.101647

20. Raza ZA, Taqi M, Tariq MR. Antibacterial agents applied as antivirals in textile-based PPE: a narrative review. Journal of the Textile Institute. 2021. https://doi.org/10.1080/00405000.2021.1889166

21. Islam MT, Sarkar C, El-Kersh DM, Jamaddar S, Uddin SJ, Shilpi JA, et al. Natural products and their derivatives against coronavirus: a review of the non-clinical and pre-clinical data. Phytother Res. 2020;34(10):2471-92. https://doi.org/10.1002/ptr.6700

22. Wang J, Wang BJ, Yang JC, Wang MY, Chen C, Luo GX, et al. Advances in the research of mechanism of pulmonary fibrosis induced by Corona Virus Disease 2019 and the corresponding therapeutic measures. Zhonghua Shao Shang Za Zhi. 2020;36(8):691-7. https://doi.org/10.3760/ cma.j.cn501120-20200307-00132 